\title{
In-Situ \& Computational Façade Performance Analysis: The B1- Campus A University Building Case in Sto. Dgo., Dom. Rep.
}

Nelson Montás Laracuente, Ph.D.

Medellin | Colombia

\author{
PUCMM| República Dominicana | arq.montas@gmail.com | nelsonmontas@uic.es
}

Marcos Barinas Uribe, M. Arq.

PUCMM| República Dominicana | barinasmarcos@gmail.com

\begin{abstract}
This paper presents experimental and simulated façade thermal and humidity performance assessments concerning three (3) types of widely used façade systems in the Dominican construction market: 8" block wall, ventilated façade \& curtain wall. Using indoor and outdoor temperature $(\Delta T)$ and humidity differences $(\Delta \mathrm{H})$ as indicators in order to compare said performances between the systems and, in turn, with environmental simulations approximating them, we try to diagnose weaknesses and foresee improvement avenues for sustainable façade systems in the Dominican context. The data was obtained by on-site measurements using eight (8) temperature and relative humidity sensors in a twelve (12) storey building in Santo Domingo, Dominican Republic.
\end{abstract}

Keywords: Façade performance, Temperature, Relative humidity, Environmental simulation, Sensors.

\section{INTRODUCCIÓN}

Ya ha sido ampliamente argumentado anteriormente que, de forma general, la envolvente arquitectónica debe ser capaz de regular y controlar, entre otros, aire, agua, luz y temperatura (Chayaamor-Heil \& Montás, 2018). Entre diversos tipos de envolventes, las fachadas sostenibles de alto rendimiento se pueden definir como cerramientos exteriores que utilizan la menor cantidad de energía posible para mantener un ambiente interior confortable, lo que promueve la salud y la productividad de los ocupantes del edificio (Aksamija, 2013). Esto significa que las fachadas sostenibles no son simplemente barreras entre el interior y el exterior; son sistemas de construcción que crean espacios cómodos respondiendo activamente al entorno externo del edificio y reducen significativamente el consumo de energía de los edificios.

Aunque el término se puede aplicar a varias acepciones, en este artículo, el rendimiento térmico se refiere a qué tan bien responde una estructura a los cambios en la temperatura externa durante los ciclos diarios y estacionales (EcoWho, 2020). Básicamente, se desea que una estructura mantenga una temperatura estable y cómoda en el interior, mientras se incurre en el menor consumo de energía posible; esto se logra mejor aumentando primero el rendimiento térmico de la estructura al proporcionar altos niveles de aislamiento (alto valor $\mathrm{R}$, bajo valor $\mathrm{U}$ ) en todas las superficies mediante la adopción de técnicas solares pasivas (EcoWho, 2020).

$\mathrm{Q}=\mathrm{UA} \Delta \mathrm{T}$

$\mathrm{Q}=$ Flujo de calor $(\mathrm{W})$
$A=$ Area de flujo de calor $\left(\mathrm{m}^{2}\right)$

$\mathrm{U}=$ Coeficiente de transferencia de calor $\left(\left[\mathrm{W} / \mathrm{m}^{2}\right]^{\star} \mathrm{K}\right)$

( $\mathrm{K}=0.93$ para vidrio corriente y $\mathrm{K}=0.856$ para aluminio)
Según Aksamija (2015), en la mayoría de los edificios, la fachada afecta el presupuesto de energía del edificio y la comodidad de sus ocupantes más que cualquier otro sistema y, siguiendo este principio, las fachadas que se implementen en climas dominados por refrigeración (cálidos) se deben tratar bajo las siguientes consideraciones:

- Control solar: protección de la fachada contra la radiación solar directa. a través de métodos de sombreado automático (forma de construcción) o dispositivos de sombreado.

- Reducción de las ganancias de calor externas: protección contra la ganancia de calor solar. por infiltración (mediante el uso de elementos de fachada opacos bien aislados) o conducción (mediante el uso de dispositivos de sombra).

- Enfriamiento: Usar ventilación natural para mejorar la calidad del aire y reducir las cargas de enfriamiento.

- Luz diurna: uso de fuentes de luz natural mientras se minimiza la ganancia de calor solar mediante el uso de dispositivos de sombra y estantes de luz. (Aksamija, 2015) 
Las simulaciones de rendimiento de edificios son una parte integral del proceso de diseño para edificios de alto rendimiento y eficiencia energética, ya que ayudan a investigar las opciones de diseño y a evaluar los impactos ambientales y energéticos de las decisiones de diseño (Augenbroe et al., 2004; Aksamija, 2009; 2010). Por extensión, son también una parte esencial del proceso de diseño para fachadas de edificios sostenibles. Predicciones cuantificables durante las diferentes etapas de un análisis forense, retroactivo, de varios tipos de fachadas es, entonces, recomendable para mejor comprensión de los aspectos de rendimiento y un mejoramiento de su diseño futuro.

El proceso de diseño ayuda a establecer métricas que se pueden utilizar para medir las mejoras mediante el uso de diferentes estrategias de proyecto. Para evaluar y optimizar el rendimiento del edificio, diferentes ciclos de análisis deberían ser parte de un proceso de diseño integrado entre herramientas de diseño CAD y de simulación (Punjabi y Miranda, 2005).

\section{METODOLOGÍA}

Para extraer e interpretar conocimiento del caso de estudio, este artículo propone un método de análisis de razonamiento inductivo-reflexivo; también llamado práctica reflexiva (Schön, 1983; Davis, 2013). Entre el 26 de octubre 2018 y el 14 de enero 2019 se realizaron ocho (8) mediciones directas continuas (en algunos casos por menos tiempo, de forma no sincrónica) en un edificio que exhibe tres (3) tipos de fachadas que se corresponden con las de una clasificación propia desarrollada previamente por Marcos Barinas, Lorianne Rosario y Nelson Montás. Como caso de estudio se eligió el edificio B1 del campus A de la PUCMM, este seleccionado por los investigadores principales dado el acceso a información relativamente sensible debido a su propiedad por parte de la universidad; habiendo seleccionado la fachada de la cara sur del mismo (por ser, a priori, la de mayor exposición al sol en un clima sub-tropical del hemisferio norte y por ende, extremadamente importante para el rendimiento -también referido aquí como performance- térmico de cualquier tipo de fachada).

Para la captura de la data se instalaron medidores (marca ELITECH, modelo GSP-6) que colectaron datos directos, in situ, sobre temperatura y humedad relativa en interiores y exteriores para así determinar un indicador de hermeticidad climática $(\Delta \mathrm{H})$ y la diferencia de temperatura $(\Delta T)$ de manera a evaluar el rendimiento de las fachadas en cuestión desde esos ángulos.

Basada en estos datos se realizó una comparación del rendimiento captado in situ de los tres (3) tipos de fachadas (façade performance) entre ellos y, a su vez, también se compararon los datos obtenidos in situ con los producidos por modelos de simulación climáticos generados en software de análisis ambiental y así se determinó un protocolo para los tipos de fachadas estudiadas con vocación a ser aplicado de manera generalizada en el contexto dominicano y posiblemente también en el latinoamericano de climas tropical y subtropical. De estas comparaciones se extrajeron conclusiones sobre su rendimiento y pertinencia en términos de diseño y construcción para su posible mejora.
Los modelos digitales de análisis ambiental presentados en este artículo fueron realizados utilizando el método de modelado parmétrico; mediante la combinación de los softwares Rhinoceros 5.0 + Grasshopper + Ladybug + Honeybee. Rhinoceros es una plataforma de diseño versátil y con una comunidad internacional inmensa (Sadeguipour \& Pak, 2013). Estos modelos están basados en archivos climáticos estándar Energy Plus Weather(EPW) que producen bases de datos llamadas Universal Thermal Climate Index (UTCI) (Mackey, 2014) que se utilizaron para determinar el estado de confort interno de las áreas modeladas en el edificio seleccionado y donde, a su vez, se corrieron simulaciones de análisis energético e irradiación solar estándar (Kaertner \& Jahn, 2016) para así realizar aproximaciones a la realidad de la construcción de los tipos de fachada ya mencionados, de manera más precisa.

\subsection{DISPOSICIÓN DE LOS SENSORES}

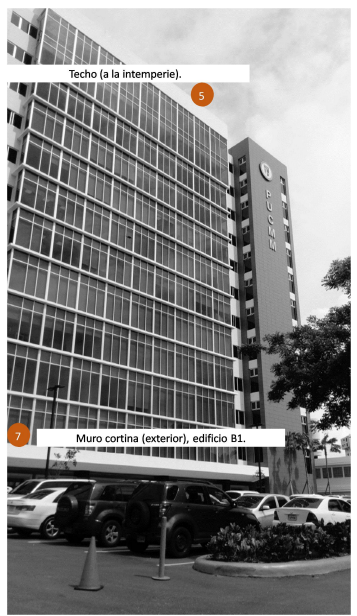

Figura 1: SENSOR 5 Techo, a la intemperie. SENSOR 7 Segundo nivel, fachada muro cortina.

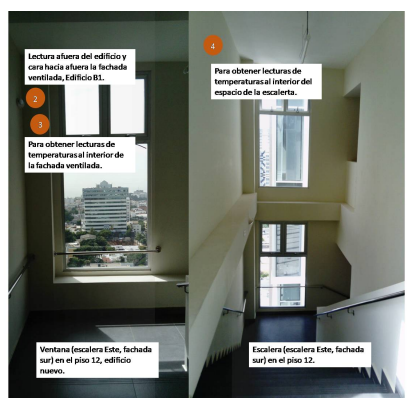

Figura 2: SENSOR 2. Lectura fuera de la fachada, paneles ventilados. SENSOR 3 Lectura dentro de la fachada, entre el panel y el muro. SENSOR 4 Lectura desde el espacio interior del edificio, fachada de paneles ventilados 


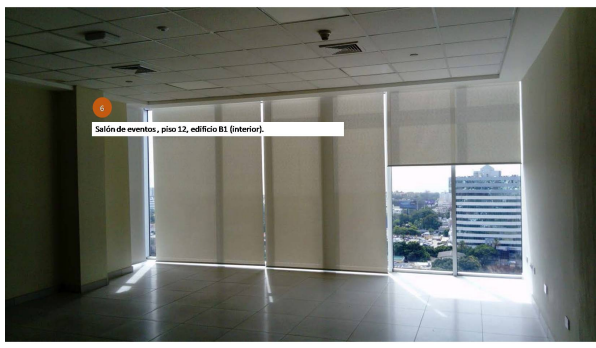

Figura 3: SENSOR 6 Salón de conferencias piso 12 (interior), muro cortina.

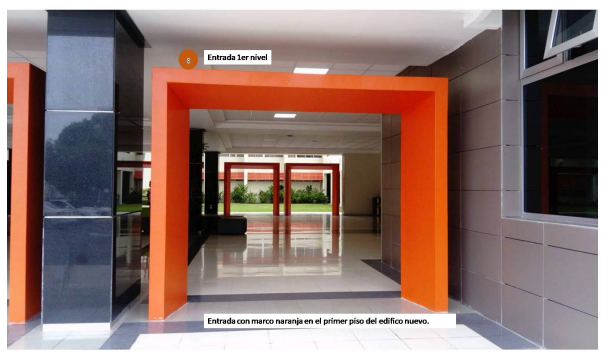

Figura 4: SENSOR 8 Entrada 1er nivel abierto bajo techo (a la sombra).

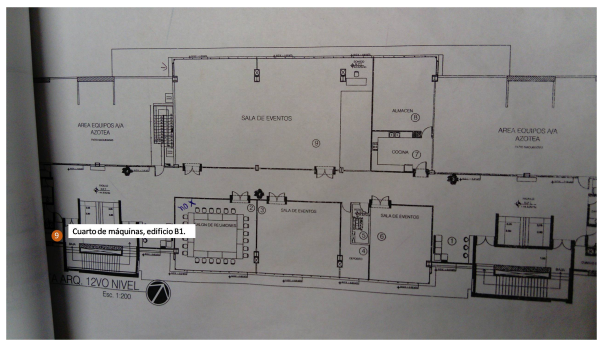

Figura 5: SENSOR 9 Cuarto de máquinas, muro de concreto.

\section{RESULTADOS:}

\subsection{LECTURAS Y COMPARACIONES DE RENDIMIENTO}

Las comparaciones mostradas en este acápite representan el rendimiento como una diferencia $(\Delta)$ con respecto a la capacidad de aislamiento térmico y de humedades de los sistemas en cuestión. El rendimiento es definido a partir de una diferencia de $C^{\circ}(\Delta T)$ cuyo valor absoluto es directamente proporcional al rendimiento del sistema en cuestión (mientras mayor sea la diferencia, mejor será el rendimiento del sistema de fachada descrito y por extensión su eficiencia energética).

\subsection{FACHADA DE BLOQUES 8"}

\subsubsection{TEMPERATURA}

Este tipo de fachada muestra una capacidad de aislamiento térmico (Figura 6), rondando a veces los $14 \mathrm{C}^{\circ}$ de diferencia $(\Delta T)$, aunque la temperatura interior se mantiene más o menos estable pero excesivamente alta para interiores (entre $30 \mathrm{C}^{\circ}$ y $32 \mathrm{C}^{\circ}$ ) - cuando la temperatura estándar de confort debería más rondar continuamente entre $22 \mathrm{C}^{\circ}$ y $26 \mathrm{C}^{\circ}$ ). Esta alta temperatura puede deberse a que el espacio en cuestión es un cuarto de máquinas que, aunque éstas que no generan calor, el espacio no está climatizado y acumule conducción de energía por algún puente térmico del techo.

\subsubsection{HUMEDAD RELATIVA}

En cuanto a su rendimiento concerniente a la humedad relativa, este tipo de fachada también muestra un buen rendimiento con una diferencia $(\Delta \mathrm{H})$ que a veces ronda el $40 \%$ con respecto a la humedad relativa exterior y manteniéndose, aunque ligeramente fluctuante, estable alrededor del $42 \%$ de humedad relativa en el interior.

\subsection{FACHADA VENTILADA}

\subsubsection{TEMPERATURA}

La fachada ventilada, en términos de temperatura es la que quizás mejor se comporta desde el punto de vista de la diferencia $(\Delta \mathrm{T})$, registrando hasta $20 \mathrm{C}^{\circ}$ con respecto a la temperatura exterior; esto gracias a que el interior ventilado del muro actúa como amortiguador. No obstante, y a pesar de la despiadada temperatura registrada en el exterior (a veces registrando hasta 47.2 $\mathrm{C}^{\circ}$ al sol), en el interior esta se mantuvo estable entre $30 \mathrm{C}^{\circ}$ y $36 \mathrm{C}^{\circ}$. Al estar localizados estos sensores en un espacio de escaleras de servicio (no de emergencia) se concluye que para mejorar el confort interior (por lo menos llevarlo a $26 \mathrm{C}^{\circ}$ o $28 \mathrm{C}^{\circ}$ ) habría que mejorar el aislamiento de la fachada (no obstante su ya buen funcionamiento), posiblemente cambiando la ubicación de las escaleras (espacio donde se registraron estas lecturas) en el diseño del edificio o gastar más energía en climatizar dicha área. 


\subsubsection{HUMEDAD RELATIVA}

Con relación a la humedad relativa, la fachada ventilada exhibe un muy buen rendimiento general, registrando a veces hasta $58 \%$ de diferencia $(\Delta \mathrm{H})$ con respecto a la humedad en el exterior, lo que atestigua buena hermeticidad en los cerramientos. Esta hermeticidad le permite mantener una humedad un poco fluctuante entre $75 \%$ y $42 \%$ según la hora, siendo las horas del día las más húmedas, especialmente luego del medio (12h) día hasta la caída del sol (17h45).

\subsection{MURO CORTINA}

\subsubsection{TEMPERATURA}

De los tres (3) tipos de fachadas estudiados, el muro cortina es el que muestra menor rendimiento térmico, desde el punto de vista de su diferencia $(\Delta T)$, cuya máxima lectura registra una diferencia de $9 \mathrm{C}^{\circ}$, relativamente bajo si lo comparamos con los otros dos sistemas evaluados anteriormente. Esto se debe, casi seguramente, a la orientación de la fachada hacia un sur casi franco unida a los materiales empleados en su construcción (vidrio, aluminio, silicona y poliestireno son el estándar) y a la ausencia de elementos de sombra directos como quiebra soles o indirectos como árboles entre otros; lo que produce un efecto de invernadero parecido al que causa el cambio climático. Esta diferencia tiende a disminuir (y por tanto su eficiencia) al avanzar la tarde. Lo que mantiene una temperatura relativamente fluctuante entre $31 \mathrm{C}^{\circ}$ y $38 \mathrm{C}^{\circ}$. Esta última súper alta para interiores, aunque cabe resaltar que el espacio estudiado (un salón de conferencias) durante este período permaneció casi durante todo el período cerrado y en desuso, por ende no climatizado. A pesar de estas condiciones de excepción, $38 \mathrm{C}^{\circ}$ sigue siendo una temperatura inadmisible en términos de confort e implica un gastp energético extremadamente alto para el sistema HVAC (aire acondicionado).

\subsubsection{HUMEDAD RELATIVA}

Con relación a la humedad relativa, el muro cortina exhibe un muy buen rendimiento general, registrando a veces hasta $60 \%$ diferencial $(\Delta \mathrm{H})$ con respecto a la humedad en el exterior (el mayor de los tres sistemas estudiados); lo que atestigua buena hermeticidad en los cerramientos. Esta hermeticidad le permite mantener una humedad un poco fluctuante entre $63 \%$ y $39 \%$ según la hora, siendo las horas del día las más húmedas, especialmente luego del medio día (12h) hasta la caída del sol (17h45).

\subsubsection{TECHO (A LA INTEMPERIE)}

\section{TEMPERATURA}

De aquí en adelante, en las tablas de este artículo se mostrarán las diferencias en $\mathrm{C}^{\circ}(\mathrm{P} \Delta \mathrm{T})$ entre las temperaturas registradas (en promedios por hora) y la temperatura de meta de $23 \mathrm{C}^{\circ}$ - escogida sobre un rango de $21 \mathrm{C}^{\circ}-24 \mathrm{C}^{\circ}$ - (Wyon, 2003); considerada en este estudio como una ideal para el confort en espacios interiores. KWH representa el esfuerzo energético debido por el sistema de climatización (HVAC) para llevar la temperatura a los citados $23 \mathrm{C}^{\circ}$. Debajo se muestran los respectivos promedios de consumo energéticos consecuentes KW por mes. Como se puede observar en las lecturas, el techo del edificio recibe los rayos del sol directamente y de manera quasi-normal (quasi-

perpendicular) (Vaisala Energy, 2020) al eje horizontal del mismo, lo que provoca temperaturas registradas diurnas de hasta $46 \mathrm{C}^{\circ}$ pero que se balancean a $21 \mathrm{C}^{\circ}$ por las noches; dejando así un promedio de $27.7 \mathrm{C}^{\circ}$ para el período estudiado. Esto descarta la hipótesis de que el techo por sí solo es el que provoca el sobrecalentamiento de la sala de máquinas y el salón de conferencias del 12vo piso, mediante la conducción bidireccional del calor y apoya una segunda suposición de que el edificio no está adecuadamente aislado y contiene el calor acumulado durante el día dentro de sí, lo que alarga y acumula temperaturas internas más altas de lo adecuado, provocando, a su vez, consumos energéticos importantes.

\subsection{COMPARACIÓN DE RENDIMIENTO EN SISTEMAS DE FACHADAS (BLOQUES 8", VENTILADA, MURO CORTINA)}

\subsubsection{TEMPERATURA}

En esta comparación de rendimientos, observamos (Figura 7) las diferencias entre cada uno de los tres (3) sistemas de fachada evaluados en varios períodos de tiempo que se solapan y permiten ver las magnitudes ejemplificadas en tándem y sacar conclusiones. Como podemos apreciar (tabla 1), en términos de temperatura, la fachada ventilada es la que mejor amortigua los cambios bruscos provenientes del exterior llegando a un máximo de $7.1 \mathrm{C}^{\circ}$ de diferencia, en promedio, seguido de cerca por el sistema de muro cortina que llega a su máximo en $8.3 \mathrm{C}^{\circ}$ para así situar, como el de menos rendimiento, al de muro de bloques de 8 " que llega a un máximo de $9.6 \mathrm{C}^{\circ}$ de diferencia con la temperatura ideal de $23 \mathrm{C}^{\circ}$

Figura 6: Mediciones sensores 2 \& 9 (fachada de bloques de 8"). Temperatura \& diferencia de temperatura en $\mathrm{C}^{\circ}$

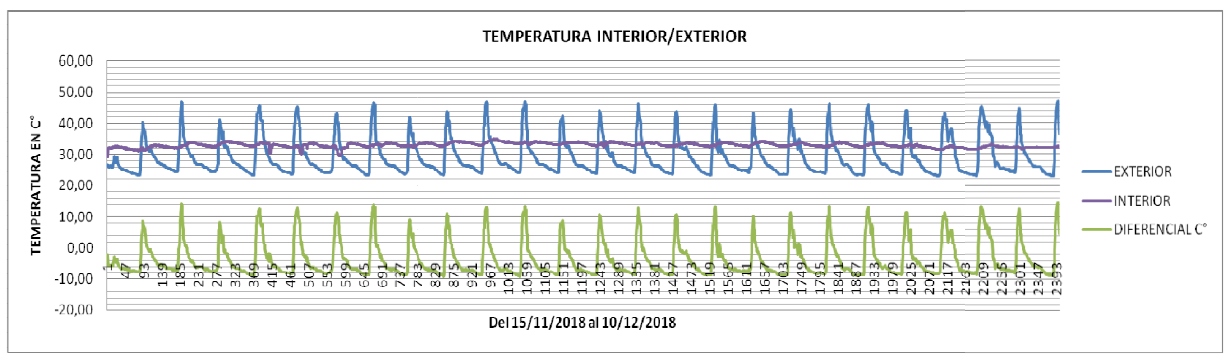




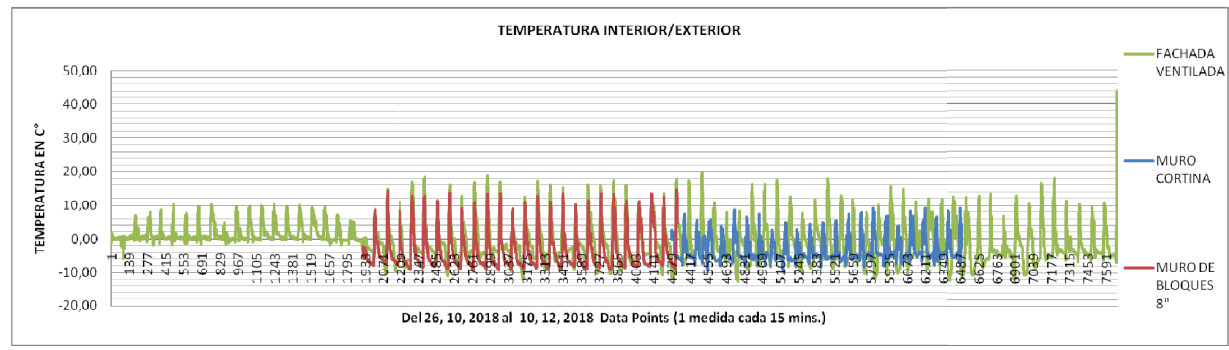

Figura 7: Comparación de diferencias entre muro cortina, fachada ventilada \& muro de bloques (8"). Temperatura en $\mathrm{C}^{\circ}$.

Tabla 1: Comparación de promedios de rendimiento entre tipos de fachada. Promedios en $\mathrm{C}^{\circ}, \mathrm{KWH}$ y mensual.

\begin{tabular}{llcl}
\hline Tipo de fachada & $\mathrm{KWH}$ & $\mathrm{KW} / \mathrm{mes}($ red.) & $\mathrm{P} \Delta \mathrm{T}\left(\mathrm{C}^{\circ}\right)$ \\
\hline Ventilada & 0,00374 & 2,47 & 7.1 \\
Muro cortina & 0,00437 & 2,89 & 8.3 \\
Bloques de 8" & 0,00506 & 3,35 & 9.6 \\
\hline
\end{tabular}

\subsubsection{HUMEDAD RELATIVA}

Con relación a la humedad relativa (Figura 8), el muro cortina exhibe el mejor rendimiento general de los tres (3) sistemas que, como podemos observar (tabla 2), registró hasta un máximo de $60 \%$ de diferencia $(\Delta \mathrm{H})$ con respecto a la humedad en el exterior, seguido relativamente de cerca por la fachada ventilada (máx. $43 \%$ ) y el muro de bloques (máx. 42\%).

Tabla 2: Comparación de promedios de rendimiento entre tipos de fachada. Humedad relativa en $(\Delta \mathrm{H}) \%$.

\begin{tabular}{ll}
\hline Tipo de fachada & $\Delta$ Humedad relativa máx. \\
\hline Muro Cortina & $60 \%$ \\
Ventilada & $43 \%$ \\
Bloques de 8" & $42 \%$ \\
\hline
\end{tabular}

\subsection{COMPARACIÓN DE SIMULACIÓN \&}

\section{LECTURAS INTERIOR SALA DE}

CONFERENCIAS (SENSOR $6,12^{\circ} \mathrm{PISO}$, EDIF. B1)

La firma de investigación estadounidense Payette Research condujo un proyecto de investigación llamado Thermal Performance of Façades (Klee \& Love, 2012) que constituye un antecedente reciente importante en los estudios comparativos entre mediciones in-situ y simulaciones digitales de eficiencia térmica y conducción de calor; estudio que fue realizado comparando, como el presente artículo, fachadas existentes construidas con modelos digitales predictivos; estos últimos realizados en el software THERM, en el cual está basado el plugin Honeybee utilizado, a su vez, en el presente estudio.

Las comparaciones mostradas aquí son un instrumento para medir la capacidad y precisión predictivas de las simulaciones en $\mathrm{GH}+$ Ladybug + Honeybee concerniendo las medidas de temperatura y humedad exteriores, conducción térmica y consumo eléctrico estimado en uno de los espacios interiores del edificio B1. Estas son modeladas como un conjunto de diferencias cuyos valores absolutos son inversamente proporcionales a la precisión (también mostrado en error porcentual) de la simulación en cuestión; o sea, mientras mayor sea la diferencia, menor será la precisión nominal de la simulación y por ende su fiabilidad predictiva.

Según Klee \& Love (2012) los sistemas construidos y los modelados por ordenador son por naturaleza imperfectos. No obstante, un modelado siguiendo las configuraciones de materiales específicas de los sistemas en cuestión, puede ayudarnos a llevar ambos, la construcción y la simulación, a una alineación más cercana (Klee \& Love, 2012). En el informe final llegaron a la conclusión de que, en promedio, los sistemas in situ mostraron un error de $50 \%$ por debajo de los modelos digitales y, en algunos casos de hasta $-70 \%$ en los sistemas más complejos (Klee \& Love, 2012) como el de cortina de cristal. Aunque el estudio en citado utilizó otro indicador como cota de referencia -el valor $\mathrm{R}$ (o sea, la resistencia térmica por $\mathrm{m}^{2}$ )- sus hallazgos parecen validarse en nuestros datos comparados (tabla 3 ) donde el error fue del $75.45 \%$ de diferencia de temperatura $(\mathrm{P} \Delta \mathrm{T})$.

\subsection{COMPARACIÓN DE CONSUMO ELECTRICO ENTRE SIMULACION \& MEDICIONES IN SITU SALA DE CONFERENCIAS (SENSOR 6, $12^{\circ}$ PISO, EDIF. B1)}

Usando mediciones in-situ de contadores de consumo de la Corporación Dominicana de Electricidad (CDDEE) \& simulaciones con $\mathrm{GH}+$ Ladybug + Honeybee (período del 9 de febrero al 11 de marzo 2019) pudimos comparar el margen predictivo de las simulaciones con respecto a la realidad: $1,584.45 \mathrm{KWH}$ (simulado) > 1,042.70 $\mathrm{KWH}$ (registrado); para una efectividad predictiva (error relativo) de $-51.95 \%$, de nuevo entre el $50 \%$ y el $70 \%$ determinado por Klee \& Love. La data muestra un margen de error contrario a lo que se esperaría ya que, si la fachada es más conductora (como lo muestran las comparaciones anteriores entre simulación y medición), el consumo energético (eléctrico en este caso) debería ser más bajo en la simulación que en las mediciones reales; el cual se muestra a la inversa del sentido lógico. Por otra parte, el error es muy significativo y la definición de las configuraciones de material y condiciones ambientales de las simulaciones deben seguirse mejorando para poder obtener predicciones más fiables en el posible diseño de sistemas de fachadas (y edificios) sostenibles. 


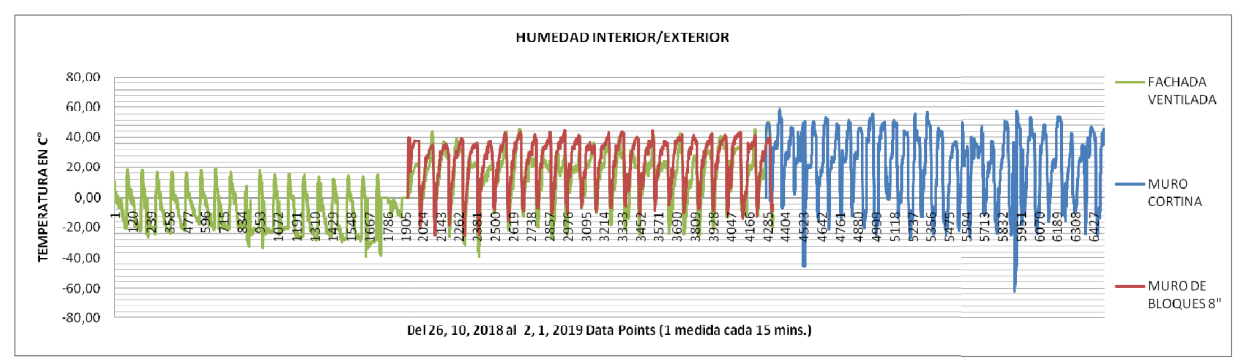

Figura 8: Comparación de diferencias entre muro cortina, fachada ventilada \& muro de bloques (8"). Humedad relativa en \%..

Tabla 3: Comparación de diferencias entre datos de simulación y lecturas de sensores, sala de conferencias (muro cortina).

\begin{tabular}{llll}
\hline Espacio simulado & $\mathrm{KWH}$ & $\mathrm{KW} / \mathrm{mes}$ (red.) & $\mathrm{P} \Delta \mathrm{T}\left(\mathrm{C}^{\circ}\right)$ \\
\hline Simulación & 0.001075 & 0.709353 & 2.04 \\
Sensor & 0.004370 & 2.884516 & 8.30 \\
Diferencia & 0.003295 & 2.175163 & 6.26 \\
DIF (\%) & 75.40 & 75.40 & 75.45 \\
\hline
\end{tabular}

Para propósitos de diseño, las simulaciones de Ladybug + Honeybee parecen ser un buen instrumento para prever posibles condiciones climáticas. No obstante, como las medidas continuas lo muestran, los promedios no aproximan, con un margen de error permisible, ni las realidades puntuales medias ni las máximas (Figura 9); por lo que se considera un instrumento, aunque útil, todavía mejorable y sus resultados deben tomarse con la cautela de que hay que compensar la falta de precisión con las medidas reales.

\section{DISCUSIÓN}

\subsection{EVALUACIÓN E INTERPRETACION DE DATOS RECOGIDOS IN SITU \& SIMULACIONES: PROMEDIOS Y PUNTOS DE DATA}

Este estudio ha determinado que, de los tres (3) tipos estudiados, la fachada de mejor rendimiento, si se toman en cuenta los promedios de $\Delta \mathrm{T}$, es el sistema de fachada ventilada. No obstante, si tomamos en cuenta los extremos de las gráficas (puntos de mayor o menor temperatura registrada), la fachada ventilada es la que registra la segunda mayor temperatura puntual ( $T$ interior de $36 \mathrm{C}^{\circ}$ ); o sea, casi la peor. Esta dualidad sugiere que, para fines de diseño, sería más juicioso tomar en cuenta ambos tipos de medidas de los datos y no sencillamente, como normalmente se hace, diseñar para el promedio ya que esto puede resultar en situaciones imprevistas e indeseables en cuanto a confort se refiere en el interior de nuestros edificios.

Una cuestión interesante resalta y es que, aunque la fachada parece ser determinante en el proceso de conducción de calor en los espacios interiores, no es la única condicionante importante en este proceso. La data colectada parece sugerir que, en el caso del edificio B1 de la Universidad, dado que los espacios interiores se encuentran en el último piso del edificio (como la sala de conferencias estudiada), el aislamiento del techo parece jugar un papel importante en el calentamiento a través del tiempo (aunque como expresado anteriormente, no así en su promedio de $\Delta \mathrm{T}$ ) generando patrones de transferencia que acumulan calor durante la noche conteniendo así la temperatura, mientras la temperatura exterior es bastante menor en magnitud.

Otro punto importante sobre el estudio es que las simulaciones, aunque con un margen de error muy mejorable, pueden ser herramientas muy importantes de previsión en las magnitudes de temperatura y humedad (especialmente cuando se toman en cuenta los promedios). Siempre y cuando se determine una desviación estadística que corrija los errores en los comportamientos estadísticos en los percentiles extremos, superiores e inferiores que es unos de los cometidos de este ejercicio. En términos energéticos, las simulaciones tuvieron una precisión de $-51 \%$ en cuanto a predicción de consumo eléctrico se refiere $(1,584.45$ $\mathrm{KWH}$ simulados >1,042.70 $\mathrm{KWH}$ registrados) lo que muestra que, obviamente, hay alguna característica del espacio en cuestión que se desconoce y esto se le puede atribuir al hecho de que el modelado de la simulación se hizo a distancia y el modelador nunca ha estado presente físicamente en el edificio en cuestión, por ende, pueden haber condiciones no aparentes en las fotos y el modelo 3D utilizado como soporte y referencia del modelo.

Figura 9: Comparación de diferencias entre datos de simulación y lecturas de sensores, sala de conferencias (muro cortina).

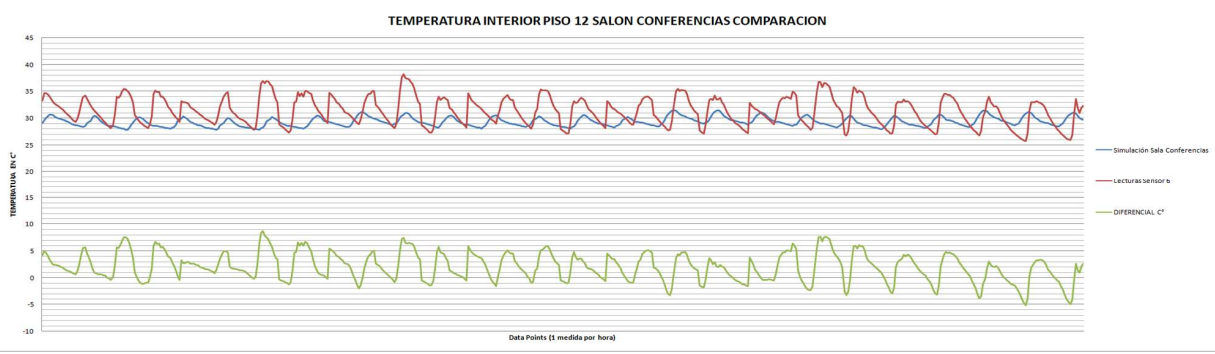




\subsection{EVALUACION DE PROMEDIOS}

\subsubsection{TEMPERATURA LECTURAS MURO CORTINA} (SENSORES 6 \& 7):

Los promedios muestran que, en la tarea de optimizar la climatización interior del espacio en cuestión, el sistema de muro cortina tiene muchos desafíos $\left(8.3 C^{\circ}\right)$ para llegar a una temperatura promedio de $23 \mathrm{C}^{\circ}$. Por lo tanto es el medio de los tres sistemas en cuanto a rendimiento.

\subsubsection{TEMPERATURA LECTURAS FACHADA VENTILADA (SENSORES 2,3 \& 4):}

El sistema ventilado, aunque es el que mayor rendimiento térmico registra de los tres sistemas, sigue siendo uno relativo bajo rendimiento, requiriendo de $7.1 \mathrm{C}^{\circ}$ de esfuerzo de la parte del sistema de climatización para alcanzar una temperatura de confort.

\subsubsection{TEMPERATURA LECTURAS MURO BLOQUES 8"(SENSORES 2 \& 9):}

El sistema de bloques de 8" es el sistema menos eficiente de los tres estudiados en términos de conducción térmica. Necesita de un esfuerzo de $9.6 \mathrm{C}^{\circ}$ en promedio para alcanzar una temperatura de confort en el interior con respecto a las registradas.

\section{APLICACIONES: BIOMIMETISMO PARA FACHADAS 3.0}

En colaboración con IN SITU Architecture, un estudio dirigido por Nicolas Vernoux-Thélot, con sede en París, estamos desarrollando una aplicación en el mundo real del trabajo analítico presentado en este artículo. Este proyecto (Figura 10), aún en curso e inspirado en la Carpinus caroliniana, consiste en una segunda piel, puesta sobre una fachada existente, que controla la luz natural y la temperatura interior en un proyecto de renovación de un edificio en París, Francia (Vernoux, 2020). El objetivo es maximizar el rendimiento térmico de la fachada, maximizando el calor interior en invierno y minimizándolo durante el verano graduando la entrada de la luz, regulando la capacidad lumínica interior y, por tanto, reduciendo el consumo eléctrico y de gas mediante el control de la temperatura; y a su vez controlando el confort interior del edificio. El desafío es diseñar un sistema de piel autorregulado de acuerdo a parámetros ambientales, térmicos y su reacción a estos. Este trabajo no sería posible sin los análisis de rendimiento térmico vehiculados en este estudio. Cabe aclarar que en este proyecto, aparte de los estudios de rendimiento térmico expuestos en este artículo, se han implementado

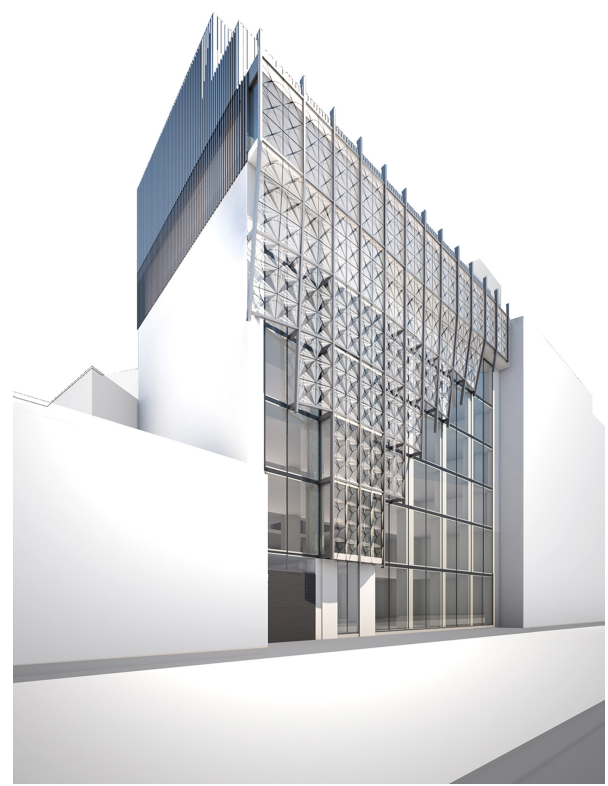

Figura 10: ๑ IN SITU Architecture 2020.

métodos adicionales de biomimética desarrollados por IN SITU Architecture (Vernoux, 2020) y de simulación de materia programable por Montás (2017).

\section{CONCLUSIONES.}

Una de las debilidades fundamentales que presenta la puesta en ejecución de estos tipos de fachadas en la República Dominicana es que los diseñadores, al concentrarse en evaluar las existencias de los sistemas disponibles, en general descuidan el aspecto de la innovación de productos. Al no proponer, dentro de sus propias metodologías la revisión, reingeniería y/o prototipado de los productos y sistemas evaluados, se quedan a la merced de las importaciones de tecnologías y materiales disponibles. Por ende, las fachadas diseñadas adolecen de conocimiento de causa embebido con relación a su rendimiento o performance, particularmente en los aspectos de física de edificación y rendimiento térmico (Figura 11) y, como resultado, los proyectos sufren sus consecuencias energéticas. 


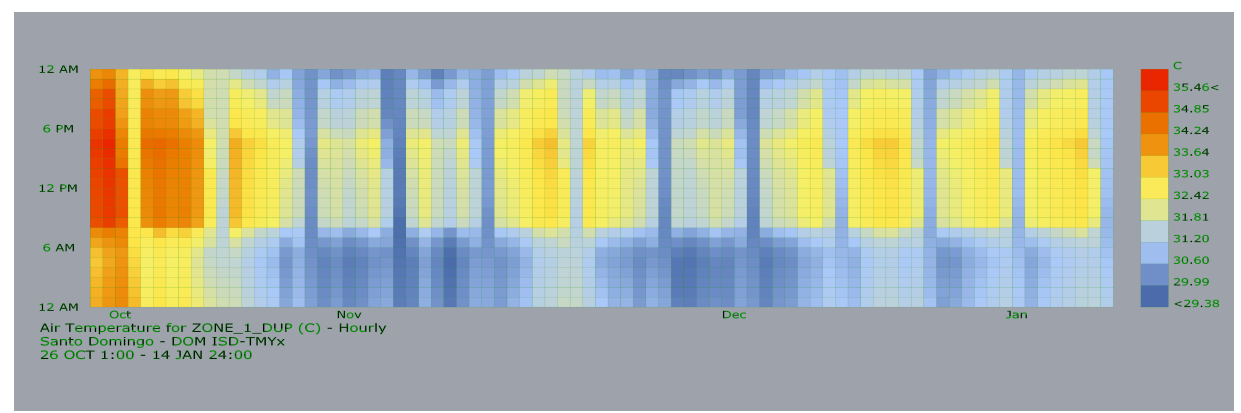

Figura 11: Simulación Ladybug + Honeybee de temperatura del aire dentro del salón de conferencias del edificio B1, Santo Domingo, Rep. Dom. Del 26 de octubre al 14 de enero.

El presente método presentado para evaluar fachadas de los tres (3) tipos descritos a lo largo de este artículo parecer tener cierto futuro en poder contribuir un paso adelante en el mencionado problema de fondo en cuanto al diseño de sistemas de envolvente en la Rep. Dom.

Todavía quedan aspectos de la formalización físicomatemática de la data recolectada que no están completamente comprendidos y cuya falta de solución obstruye nuestra capacidad de predecir y alinear con exactitud el comportamiento de sistemas en configuraciones de múltiples materiales (cada uno con un valor $\mathrm{R}$ diferente) con las medidas in situ; aspectos que están en proceso de análisis y comprensión.

\subsection{TRABAJO FUTURO}

Como fase próxima, la idea es de establecer e implementar, dados los datos recolectados acá, una metodología de diseño utilizando el modelado paramétrico y el prototipado para formalizar, en el marco de experimentos controlados de laboratorio, un proceso que integre las lecciones aprendidas de los experimentos aquí mostrados en vista a mejorar las aproximaciones predictivas iterando el método de evaluación anteriormente descrito para su implementación en el diseño de fachadas más eficientes. De esta manera la selección del sistema, los materiales empleados y su disposición arquitectónica, se especula, serán más adecuados.

\section{REFERENCIAS}

Aksamija, A. (2009). Integration in architectural design: methods and implementations. Design Principles and Practices: An International Journal, Vol. 3, 151-160

Aksamija, A. (2013). Sustainable Facades: Design Methods for High-performance Building Envelopes (1st ed.). Hoboken, NJ: John Wiley \& Sons.

Aksamija, A. (2010). Analysis and computation: sustainable design in practice. Design Principles and Practices: An International Journal, Vol. 4, 291-314.

Aksamija, A. (2015, July). Sustainable facades: design methods for Energy Efficient Facades, Building Innovation Research Knowledgebase (BIRK), Washington, D.C. American Institute Of Architects (AIA).

Augenbroe, G., de Wilde, H., Moon, J., \& Malkawi, A.. (2004). An interoperability workbench for design analysis integration. Energy and Buildings, Vol. 36, 737-748.

Davis, D. (2013). Modelled on Software Engineering: Flexible Parametric Models in the Practice of Architecture, School of
Architecture and Design College of Design and Social context, RMIT University.

Connor, N. Thermal engineering.org, What is Heat Exchanger Heat Transfer Coefficient - U-Factor - Definition, 2019, Retrieved from: https://www.thermal-engineering.org/what-isheat-exchanger-heat-transfer-coefficient-u-factor-definition/

EcoWho (2020). Thermal Performance. Retrieved from: https://www.ecowho.com/defn/t/thermal+performance/3e67d

Heil, N., \& Montás, N. (2018). Biomimetic Building skin: Living Envelope for Contemporary Architecture. In Quique Zarzo (ed.), The Power of Skin: New materiality in Contemporary Architectural Design (chapter 13). ETSAM, Universidad Politécnica de Madrid: Arcadia Mediática, Lima, Perú.

Kaertner, A, Jahn, C. (2016) C., Ladybug Tutorial 4.41. Retrieved from: http://www.ia.arch.ethz.ch/wp content/uploads/2015/11/4.411-LadybugTutorial_SRadiation.pdf

Mackey, C. (2014) Ladybug Comfort Tutorials - Outdoor Comfort: UTCl. Retrieved from https://www.youtube.com/watch?v=hwErNMWeQVw

Montás, N., Baquero, P. \& Giannopoulou, E. (2017). Parametric Modeling Implementation for Kinetic Systems Simulation: Programmable Matter Matters, in Estévez, Alberto (ed.), Proceedings from the 3 rd International Conference on Biodigital Architecture \& Genetics (pp.10-12) Universitat Internacional de Catalunya, 2-5 June, Barcelona, Spain

Klee, C. \& Love, A. (2012). Thermal Performance Of Façades, 2012 AIA Upjhon Grant Research Initiative. Fayette Research, 290 Congress St., Fifth Floor, Boston, MA, USA Retrieved from: https://www.payette.com/wpcontent/uploads/2017/09/2012_aia-upjohn-grant_thermalperformance-of-facades payette-final-report.pdf

Sadeguipour, M.R. \& Pak, M. (2013). Ladybug: A Parametric Environmental Plugin for Grasshopper to Help Designers Create an Environmentally-conscious Design. Proceedings of the 13th Conference of the International Building Performance Simulation Association, Chambéry, France, August 26-28. Retrieved from: http://www.ibpsa.org/proceedings/bs2013/p_2499.pdf

Schön, D. (1983). The Reflective Practitioner: How Professionals Think in Action. London: Maurice Temple Smith.

Vaisala Energy (2020) What is Direct Normal Irradiance? Retrieved from https://www.3tier.com/en/support/solar-onlinetools/what-direct-normal-irradiance-solar-prospecting/

Vernoux, T. (2020, July). Quand l'architecture imite la nature. CNRS Le journal, January. Retrieved from: https://lejournal.cnrs.fr/billets/quand-larchitecture-imite-lanature.

Wyon, D.P. (2003). Thermal Environmental Effects on Performance. In J. Spengler, J.M. Samet \& J.F. McCarthy (Eds.), IAQ Handbook: chapter 16 (pp. 353-368). New York, NY: McGraw-Hill. 